\title{
An Open-Access Web-based medical image atlas for collaborative Medical image sharing, processing, Web Semantic searching and analysis with uses in medical training, research and second opinion of cases
}

Atlas Web de acceso abierto de imágenes médicas para compartir, analizar, procesar y buscar en Web Semántica imágenes médicas con aplicaciones en entrenamiento, investigación y segunda opinión de casos en salud

Jorge Millan ${ }^{1}$, Leonardo Yunda ${ }^{1}$

\begin{abstract}
Objective: Development of a Web-based atlas for collaborative image sharing, processing and analysis of diagnostic images. Materials and methods: Use of Web 2.0 Personalized Learning Environment tools for social learning and knowledge construction and sharing. Results: The platform allows registered users to upload, visualize, process and comment medical images in a collaborative manner. The system contains a social network module for open case discussion, a video conference and webinar module for real time case analysis, and an image visualization, annotation and processing module for image analysis. The developed open-access platform serves as a large community-created and validated free-repository of diagnostic medical images to be used for training, research as well as reference and second opinion of cases.
\end{abstract}

Keywords: medical image atlas, image database, DICOM, medical social Web.

\section{Resumen}

Objetivo: Desarrollo de un atlas Web para el intercambio colaborativo, procesamiento y análisis de imágenes diagnósticas. Materiales y métodos: Uso de herramientas Web 2.0 para desarrollo de Ambientes de Aprendizaje Personalizado para el aprendizaje social y la construcción e intercambio del conocimiento. Resultados: La plataforma permite subir, visualizar, procesar y comentar las imágenes médicas de forma colaborativa. El sistema posee un módulo de red social, módulo de Webinars para análisis de casos en tiempo real, y módulo de visualización, anotación, análisis y procesamiento de imágenes. El sistema tiene usos como un gran repositorio de imágenes diagnósticas creado y validado por la comunidad médica y utilizado para la formación e investigación, así como para la referencia y segunda opinión de casos.

Palabras clave: atlas de imágenes médicas, base de datos de imágenes, DICOM, Web social en salud. 


\section{Introduction}

Remotely accessing and visualizing medical images through the web is being implemented for training, research and expert consultation activities in medical imaging. With a web browser, healthcare professionals can access and browse medical images from anywhere at any time, via different devices (computer, mobile device) (1), without installation and maintenance of additional client software. Remotely accessing images helps to increase the productivity of healthcare professionals and gives a more efficient solution to teleradiology and medical image data sharing systems (2). As communication and information technologies have evolved, tools and technologies for dynamically accessing and sharing knowledge over the Web have increased, allowing the development of systems adequate for ubiquitous online and offline training, collaborative research and asynchronous and synchronous case discussion for increased knowledge generation and transfer.

With the widespread use of the Internet, the demand for accessing and visualizing medical images via the Web has increased in recent years mainly due to the success and introduction in clinical use of Telehealth applications such as Teleradiology and Teleconsultation in remote areas. Most medical imaging devices support DICOM (digital imaging and communications in medicine) format (3), which is the widely accepted standard for medical image storage and delivery among medical devices. DICOM images with different modalities (e.g. CT (computed tomography), MR (magnetic resonance), DX (digital radiography), PET-CT (positron emission tomography CT) X-ray, etc.) can be managed by a health information system. PACS (picture archiving and communication system in medicine) is the widespread system for DICOM image management and presentation within a hospital and radiology center. However, PACS systems are expensive, which limits its widespread use in low budget clinics and hospitals worldwide.
As a result of the advances of the Web as well as information and communication technologies, multiple Web sites have emerged to allow access to medical images remotely through a Web browser (4-6), enabling users to access medical images from home and office, without specialized computer platforms or software. Some of these sites offer medical image atlas $(7,8)$ specialized to a clinical application such as dermatology (9), ultrasound (10-11), malaria (12) and general radiology $(13,14)$ where the user is not an active participant of the comments or image sharing process but merely a viewer. These sites have been developed to be used as reference sources for teaching and research (15). More recent developments have included social media image sharing $(16,17)$, semantic image and content based searching techniques (18-22) as well as support systems for evidence based medicine (23). Other image database applications include telemedicine $(24,25)$, online teaching in nursing $(26,27)$ and medical image analysis (28-29).

These online medical image databases until now have supported only basic functions, such as static image display and content search. The available atlases are specific to a clinical specialty and do not combine multiple diagnostic imaging modalities. Other limitations of current systems are the lack of online processing functions which would be ideal for development and validation of new image processing algorithms.

Our developed system provides more versatile and flexible access and manipulation of medical images. It supports a number of interactive multimedia operations, such as online drawing and annotations, online image processing, semantic content search and image display manipulation functions. Because of the ability of users to upload, comment and rate on medical images, the developed system can be augmented and enriched by the healthcare community adding knowledge creation and transfer in a social networking environment. The 
system uses rapid development concepts such as those based on open-source libraries which have proven effective in the development of medical imaging visualization tools (30).

\section{Personalized Learning Environment PLE in Healthcare}

Healthcare professionals are continuously in need of training and education. However, distance, time and cost of training in the traditional educational setting makes it costly, not dynamic and not always convenient. Fortunately, recent Web 2.0 tools can make the educational process interactive, with social network sites facilitating collaboration between people. Web 2.0 technologies and tools in the Web allow interaction between users and construction of knowledge in the social network environment. Personal learning environments (PLE) help learners take control of and manage their own learning. A Web 2.0 site with social network capabilities is expected to provide visitors with the environment to actively participate in the knowledge generation and transfer process.

Technology-enhanced learning in healthcare education spans instructivist to constructivist approaches, as shown in the E-learning ladder diagram of Moule $(26,27)$. Instructivist learning theory is a teacher- centered model of learning. In constructivist theory the student constructs new and relevant knowledge through analysis of information and reference to experience and understanding. The knowledge the student constructs is pertinent and customized to his/her needs and interests. Opportunities for social learning and the construction of knowledge, where learners are active in creating their own knowledge and understanding, can be offered through Web 2.0 tools, discussion boards and other social networking sites. As shown in Figure 1, Web 2.0 tools, present a range of technologies with the potential to enhance learning and teaching, and make the educational process interactive, dynamic and collaborative at a reduced cost.

Using Web 2.0 tools and the model of constructivist learning, which has great potential for effective online and personal learning in healthcare, the developed platform in this work incorporates PLE elements for social learning such as video conference, desktop sharing, social network, as well as image commenting and sharing tools. The goal of the developed platform is to serve as an online educational community to discuss medical cases and construct knowledge, which can lead to increased medical knowledge, evidence based medicine and ultimately, better diagnosis and healthcare service.

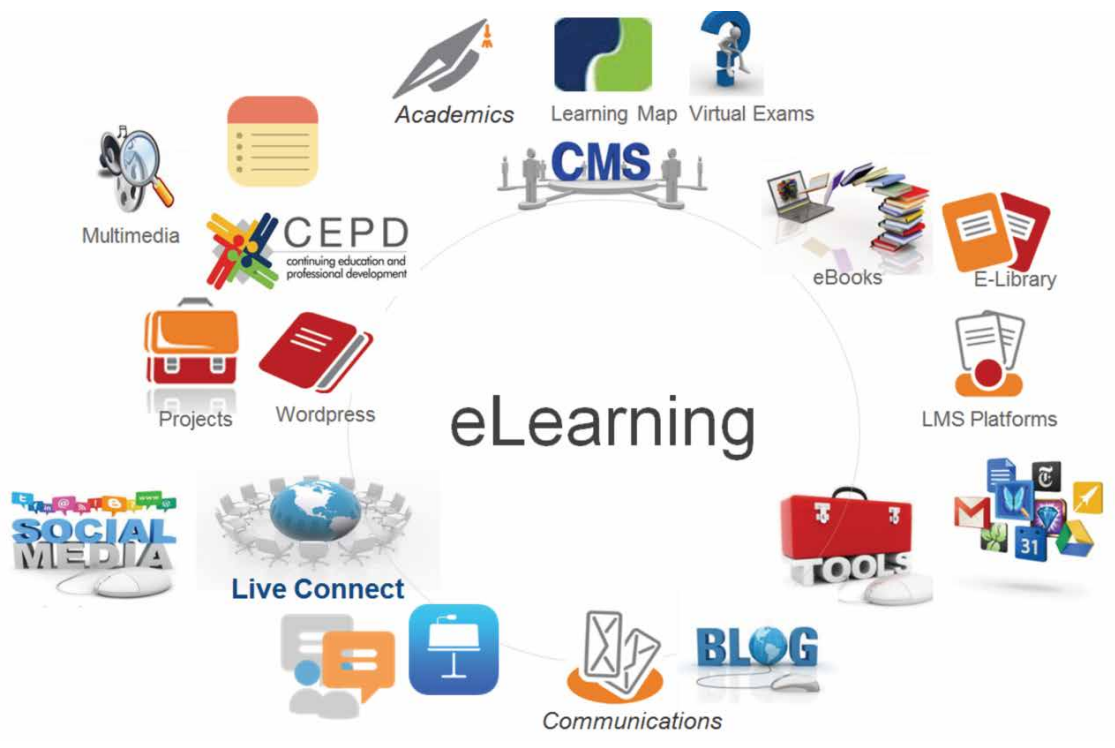

Figure 1. E-learning tools and technologies. 
Potential uses of the medical image atlas include uses as a reference source for case discussion and second opinion, for interconsultation, for research and discovery of features and disease signatures embedded in images, and as an image database to develop and validate new image processing algorithms.

\section{Materials and methods System Description}

The developed web-based medical image atlas implements the following major modules: 1) User management, 2) Graphical User Interface,
3) Image and video upload and visualization, 4) Image manipulation, 5) DICOM viewer, 6) Social networking, and 7) Video conference. The web site has been developed in PHP using MyS$\mathrm{QL}$ as the server database. Other programming technologies include JavaScript and JQuery plugins. To reduce development time and to benefit from open-source stable developments, various third party plugins have been used: A plugin for DICOM images, a plugin for video conference and a plugin for image manipulation. Figure 2 shows the system architecture for the developed medical image atlas.

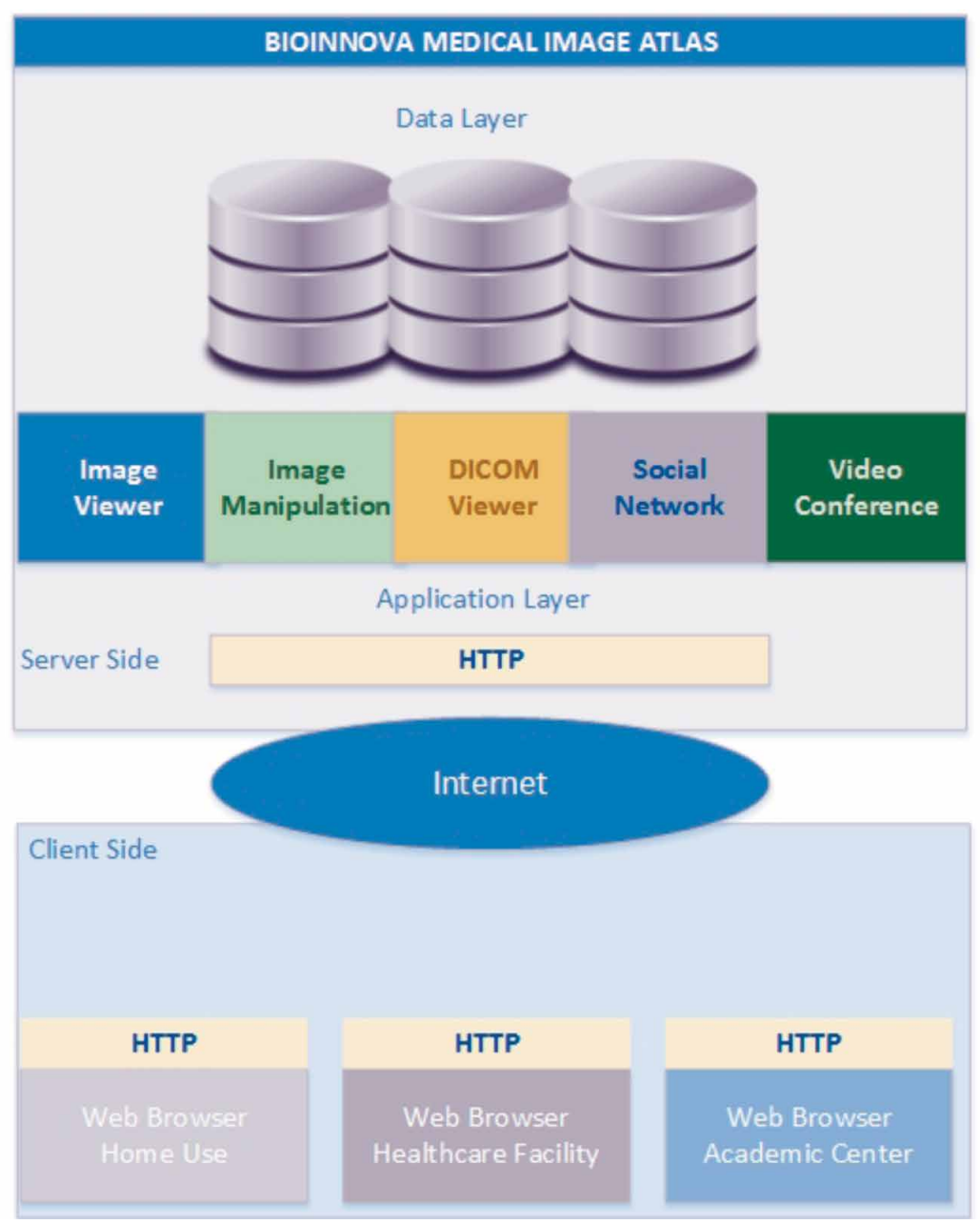

Figure 2. System diagram for the Web-based Medical Image Atlas with image access and manipulation. The Web browsers at the client side execute the application modules on the user's PCs. 
The medical image Web server consists of a data layer and an application layer. The data layer supports multiple interfaces for connections to medical images. The application layer consists of five modules - online image viewer, online interactive image manipulation, DICOM viewer, social network and Videoconference.

\section{User Interface}

The Web-based system Graphical User Interface (GUI) is as shown in Figure 3. Images and videos are displayed in a rectangular paged array of thumbnails for easy image loading and visualization. User interface controls include a search box, command buttons to select recent images, commented images, favorites and featured images. It has a dropdown selector to display the type of image according to the diagnostic image type classification. The GUI has a command button to upload and visualize DICOM images. Additional interface icons include login, and member profile areas. A Google hangout function icon implements group videochat and desktop sharing for asynchronous communication and real time collaboration and case discussion

In the medical image viewer module, the visitor can search medical images using metawords such as study type and disease conditions. The system also has a semantic search module for content based searching. From the query results obtained, the selected image data set is viewed and opened using the online image manipulation module, $\mathrm{Fi}$ gure 4 . The image is initially shown in thumbnail format; clicking on that initiates the display of the full-resolution image in the main display window.

\section{Image Manipulation Module}

The image manipulation module is based on the Aviary photo editor plugin accessible at http:// www.aviary.com site. The Aviary photo editor is a freely available web plugin that implements image manipulation functions on a selected image. Functions include drawing pencil with color palette, text annotations, image resize, cropping, brightness, contrast and saturation.

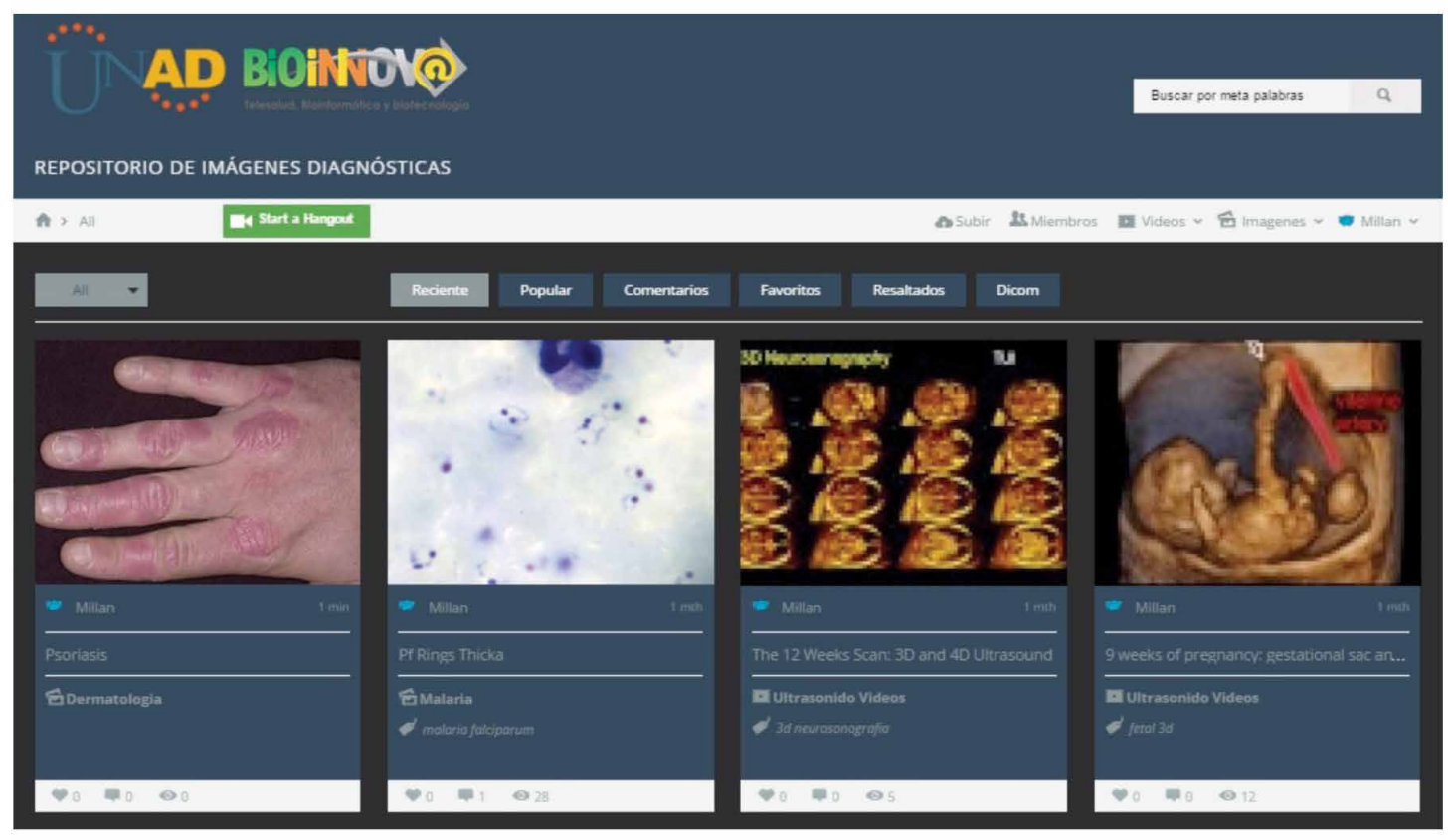

Figure 3. Graphical User Interface illustrating thumbnail images and command buttons. 


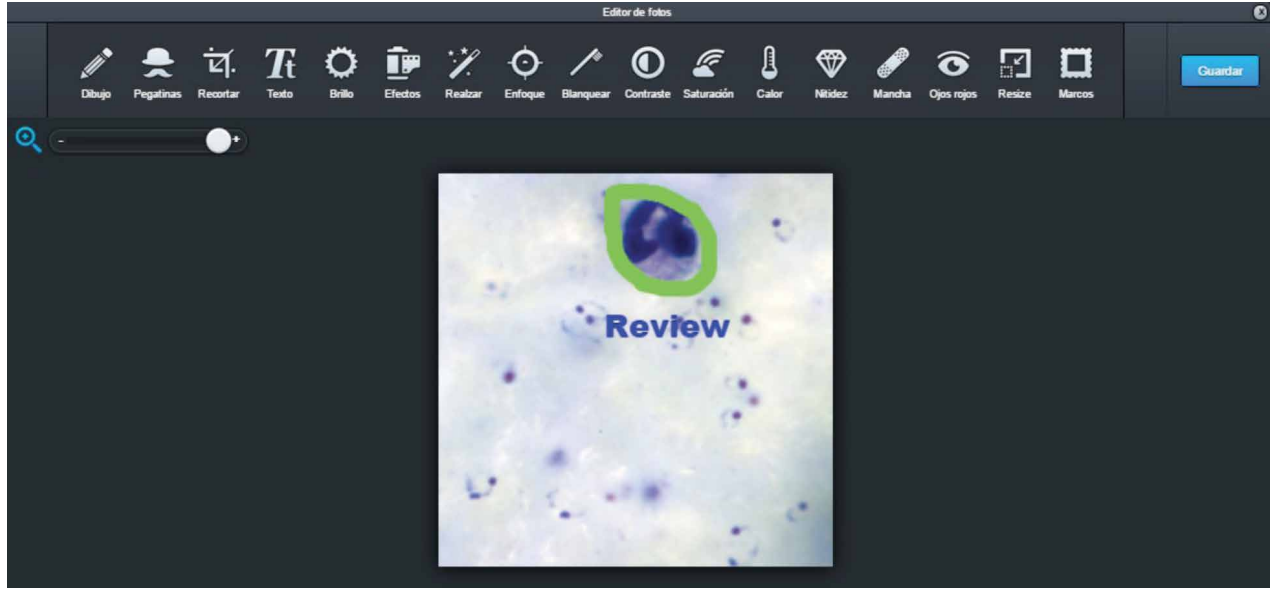

Figure 4. Image visualization module. Top icons implement image manipulation and processing functions.

The image module also includes various pre-defined image enhancement functions which can be used to improve the visualization and detection of image features. Platform users can view and manipulate images and compare the effect of the various image enhancement functions on the selected medical image for better feature and object visualization and analysis. Figure 4 shows the interface for the image manipulation module.

\section{DICOM Viewer Module}

The DICOM viewer module is implemented integrating into the platform a web plugin that allows opening, visualization and processing of DICOM medical images. The integrated plugin is a freely accessible module found at http://ivmartel.github.io/dwv/. The plugin is a simple JavaScript/HTML5 DICOM Web Viewer, which can be used on any platform that provides a modern browser (laptop, tablet, phone and even modern TVs), without the need to install any additional software.

The DICOM viewer implements some basic processing and annotation functions on the uploaded image such as drawing, text annotation, threshold filtering, zoom/pan and scroll. It also allows viewing the DICOM image header with demographics and conditions for image acquisition. Users of the system can upload, visualize and manipulate DICOM images obtained from various medical image acquisition devices such as ultrasound, CT and MRI equipment.

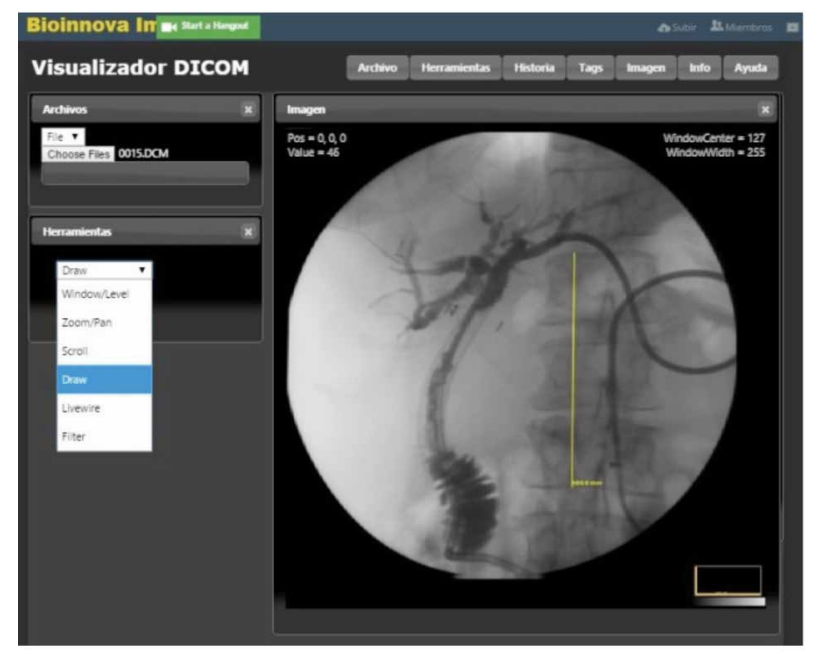

Figure 5. DICOM viewer module illustrating a DICOM image and basic image manipulation tools.

The DICOM module supports a number of common image processing functions, including ROI operations, image smoothing and enhancement, and variable display intensity. The ROI functions include

1. Common rectangle, ellipse, and shape (freehand drawing of any region) ROI drawing and calculation

2. Text annotation and labeling

3. Distance measurement, and

4. Color selecting for drawing pen or text.

5. Histogram window 


\section{Color transformation \\ 7. Filter: Sharpen and Sobel}

The online medical videoconference module provides tools for consultations with other physicians at remote sites, or for case discussion in clinics and academic centers. It supports online discussion and desktop sharing for simultaneous image viewing and analysis. Interactive drawing and annotation on the image are displayed in real time. The online discussion chat lets physicians consult with each other, add clinical descriptions and comments, and implement healthcare training and education.

\section{Summary of features:}

- User Registration and Member Area

- Image uploader and viewer

- Semantic image and content based search

- Annotation and Comments

- Image Processing and Analysis

- Dicom Viewer and Image Processing

- Social Media and Image annotations

- Webinar/Videoconference

- Video Embed and Video Library

- Upload and Share Slides

\section{Conclusions}

The developed Web-based interactive medical image atlas system provides efficient and flexible digital medical image data access and manipulation over the Internet, thus offering great benefits for training, research, as well as for consultation and second opinion of cases. It has the potential to become a community created reference source of diagnostic images from multiple medical imaging modalities. An example of its potential application is the expert evaluation of images acquired at remote sites in rural areas, which often lack of local specialist services.

Future developments include the ability to run scripts and digitally process medical images for research and development of new image processing methods. Future work also includes Interactive $3 \mathrm{D}$ image data presentation modules and content based searching techniques, which will further enhance the medical image atlas system. A community created document library including videos, eBooks and slide presentations for training and education can be implemented in future releases.

\section{Acknowledgement}

This research has been supported by the School of Health, Universidad Nacional Abierta y a Distancia UNAD, Bogota, Colombia

\section{References}

1. Dragan D, Ivetic D. Request redirection paradigm in medical image archive implementation. In: Computer Methods and Programs in Biomedicine. 2012; 107: 111-121.

2. Kotter E, Baumann T, Jäger D, Langer M. Technologies for image distribution in hospitals. In: European Radiology. 2006; 16:1270-1279.

3. DICOM, Digital Imaging and Communications in Medicine, ACR (the American College of Radiology) and NEMA (the National Electrical Manufacturers Association), http://medical.nema.org/standard.html

4. Dayhoff R. A Medical image database system. In: Computers in Healthcare. 1988; 9 (12): 39.

5. Weidong C, Dagan F, Fulton R. Web-Based Digital Images. In: IEEE Computer Graphics and Applications. 2001: 21(1): 44-47.

6. Shena H, Maa D, Zhao, et al, MIAPS: A web-based system for remotely accessing and presenting medical images. In: Comput Methods Programs Biomed. 2014; 113(1):266-83

7. Armato S., McLennan G, et al. Lung Image Database Consortium: Developing a Resource for the Medical Imaging Research Community. In: Radiology, 2004 Sept; 22(3): 739-48.

8. King C. Images. MD-The Online Encyclopedia of Medical Images. In: Journal of Electronic Resources in Medical Libraries. 2009; 6:163-168.

9. http://www.dermnet.com/

10. http://www.ultrasoundcases.info/

11. http://www.ultrasound-images.com/

12. US Centers for Disease Control and Prevention. http://www. cdc.gov/dpdx/malaria/gallery.html\#pfalringformtrophs

13. National Institutes of Health, National Cancer Institute, National Biomedical Imaging Archive accessible at https:/imaging.nci.nih.gov/ncia/login.jsf

14. Clark K, Gierada D, et al. Creation of a CT Image Library for the Lung Screening Study of the National Lung Screening Trial. In: Journal of Digital Imaging. 2007; 20(1): 23-31. 
15. National Institutes of Health, Center for Information Technology, MIPAV. http://mipav.cit.nih.gov/

16. Niinimäki M1, Zhou X, De la Vega E, Cabrera M, Müller H. A web service for enabling medical image retrieval integrated into a social medical image sharing platform. In: Stud Health Technol Inform. 2010; 160 (Pt 2): 1273-6.

17. Hong S H, Lee S J. An Intelligent Web Digital Image Metadata Service Platform for Social Curation. In: Modelling and Simulation in Engineering. Article IS 651428. In Press 2014

18. Stathopoulos S, Kalamboukis T. Applying latent semantic analysis to large-scale medical image databases. In: Comput Med Imaging Graph. 2015; 39:27-34.

19. Tagare H, Jaffe C, Duncan J, Medical Image Databases: A Content-based Retrieval Approach. In: Journal of the American Medical Informatics Association. 1997; 4(3):184-98

20. Park S C, Sukthankar R, et al. Optimization of reference library used in content-based medical image retrieval scheme. In: Med. Phys. 2007; 34(11):4331-4339

21. Turinsky A, Stromer J, et al. Integration of Genomic and Medical Data into a 3D Atlas of Human Anatomy. In: Stud Health Technol Inform. 2008; 132:526-31

22. Mendi E, Bayrak C, et al. Content-Based Management Service for Medical Videos. In: Telemedicine and e-Health. January 2013; 19(1):36-41

23. Wang K, Jeanmenne A, et al, An Online Evidence-Based Decision Support System for Distinguishing Benign from Malig- nant Vertebral Compression Fractures by Magnetic Resonance Imaging Feature Analysis. In: Journal of Digital Imaging. 2011; 24(3):507-515.

24. Hasselberg M, Beer N, et al. Image-Based Medical Expert Teleconsultation in Acute Care of Injuries. A Systematic Review of Effects on Information Accuracy, Diagnostic Validity, Clinical Outcome, and User Satisfaction. In: PLoS ONE. 2014; 9(6): 1-16.

25. Venkatram N, Reddy L., et al. RSA-DWT Based Medical Image Watermarking for Telemedicine Applications. In: Journal of Theoretical and Applied Information technology. July 31, 2014: 65 (3): 801-813.

26. Moule P, Ward R, Lockyer L. Nursing and healthcare students' experiences and use of e-learning in higher education. In: Journal of Advanced Nursing. 2010; 66(12):2785-2795

27. Moule P, E-learning for healthcare students: developing the communities of practice framework, 370. In: Journal of Advanced Nursing. 2006; 54(3):370-380.

28. Baltasar A, González-Sistal A. Design of a Web-Tool for Diagnostic Clinical Trials Handling Medical Imaging Research, Journal of Digital Imaging. April 2011; 24(2):196-202.

29. Wollny G, Kellman P, et al. MIA - A free and open source software for gray scale medical image analysis. In: Source Code for Biology and Medicine. 2013; 8:20.

30. Caban J., Joshi A. and Paul N. Rapid Development of Medical Imaging Tools with Open-Source Libraries. In: Journal of Digital Imaging. 2007; 20(1): 83-93. 